\title{
"Handbook of biomedical optics", edited by David A. Boas, Constantinos Pitris, and Nimmi Ramanujam
}

\author{
Boris Gramatikov
}

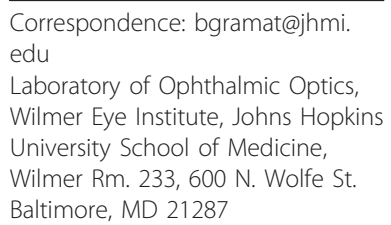

Correspondence: bgramat@jhmi. edu

Laboratory of Ophthalmic Optics, Wilmer Eye Institute, Johns Hopkins University School of Medicine, Wilmer Rm. 233, 600 N. Wolfe St. Baltimore, MD 21287

\author{
Abstract \\ David A. Boas, Constantinos Pitris, and Nimmi Ramanujam, Eds.: \\ Handbook of Biomedical Optics \\ CRC Press, Taylor and Francis Group, Boca Raton, London, New York, 2011 \\ ISBN: 978-1-4200-9036-9 (Hardback), 787 pages
}

Biomedical Optics is becoming increasingly important to medical applications. Its multidisciplinary knowledge has been expanding steadily and exponentially in the last few decades. Yet, despite the publication of a significant number of books in the field in recent years, there is a growing need of an extensive, all-encompassing reference book. The publication of Handbook of Biomedical Optics could, in my view, be considered a successive attempt in this direction.

The book consists of 35 chapters organized into six parts.

Part I contains introductory material on optics and optical properties of tissues. Although most of the material presented here can be found in basic books on geometrical optics, this part is a unique compendium of theoretically sound, well illustrated and directly applicable knowledge. I like the use of the matrix method in paraxial optics, which allows elegant formalization, as well as efficient implementation of geometrical optics in MATLAB models. Basic concepts such as electromagnetic waves and their motion, diffraction optics, refraction, reflection and transmission are explained briefly and yet precisely. In addition to light sources and detectors, issues like photobleaching, phototoxicity and thermal damage are discussed, and irradiation guidelines are presented. Particularly useful I find the chapter on optical properties of biological tissues (chapter 5), which gives pretty exhaustive data on absorption, transmission, reflectance and scattering of tissues from different organs at different wavelengths. Given that only a very few literature sources offer such data, I find this information valuable, especially for laser surgery and photodynamic therapy.

Part II deals with spectroscopy and spectral imaging, as applied to biology and medicine. Of particular interest here are methods exploiting intrinsic absorption and scattering contrast, dynamic contrast, Raman contrast mechanisms, broadband diffuse optical spectroscopic imaging, hyper-spectral imaging, near-infrared diffuse correlation spectroscopy for measuring tissue blood flow, and fluorescence spectroscopy. In

(c) 2012 Gramatikov; licensee BioMed Central Ltd. This is an Open Access article distributed under the terms of the Creative Commons Attribution License (http://creativecommons.org/licenses/by/2.0), which permits unrestricted use, distribution, and reproduction in any medium, provided the original work is properly cited. 
addition to the theoretical breadth and depth, numerous practical examples are given, and plenty of validation work is cited. Among the many topics and applications included here, I found the chapters on light scattering spectroscopy and broadband diffuse optical spectroscopic imaging particularly exciting. The former (chapter 8) is centered on an excellent introduction to the authors' original principle and device for confocal light absorption and scattering spectroscopic (CLASS) microscopy, capable of assessing epithelial cell morphology for performing pathology in vivo and real-time and thus, unlike biopsy, allowing continuous monitoring as pathology progresses. The other application that caught my attention (chapter 9) is a functional imaging technique acquiring macroscopically averaged tissue absorption and scattering parameters at depths up to several centimeters, based on a combination of time-independent continuous-wave (CW) NIR spectroscopy with frequency-domain photon migration (FDPM) technology. Although developed and tested mainly for breast cancer detection, this technique allows continuous monitoring of the effects of chemotherapy in breast tumors in a non-invasive and non-ionizing manner. Further, chapter 12 gives intimate knowledge on highly chemically specific techniques such as Raman scattering and midinfrared absorption.

Part III focuses on tomographic imaging method. It brings the reader up to speed in many topics related to optical coherence tomography (OCT) and other tomographic techniques. The material on OCT is up-to-date and systematic, and references to the nearly 200 most important publications in the field in the last twenty years is given in chapter 13 alone (Introduction and Theory). This chapter is likely challenging for those without a significant background in mathematics and basic physics. In contrast, chapter 14 on functional OCT in preclinical models is easy to comprehend, conveying interesting ideas and providing important, often first-hand knowledge on hemodynamic imaging of microvessel blood flow with Doppler OCT, or alternative techniques, such as single-pass volumetric bidirectional flow imaging (SPFI), single-pass volumetric bidirectional flow imaging combined with spectral domain OCT (SPFI-SDOCT), and speckle variance OCT. A good deal of clinical applications of OCT in ophthalmology, cardiology, gastroenterology and other areas is given in chapter 15. Chapter 16 deals with forward models of light transport in biological tissue, but, again, may turn out to be a challenge for the ones lacking solid background in math and physics. For the rest of us, it will be a useful tool for computer modeling of biological tissues using numerical methods. The same can be said of chapter 17 (Inverse Models of Light Transport), in which this reviewer, however, did not find much, if any, examples of optical tomography reconstruction of biological tissue samples. Chapters 19 and 20 are focused on diffuse optical tomography (DOT) using continuous wave (CW), frequency domain (FD), or time domain (TD) methods. Based on systematically presented theory of modeling light propagation and image reconstruction, these chapters describe useful applications of DOT to in vivo imaging in breast cancer, brain tissue, joints (for rheumatoid arthritis), prostate etc. Chapter 21 provides insight into the exciting emerging field of photoacoustic tomography and ultrasound-modulated optical tomography - two hybrid imaging modalities combining acoustic and optical phenomena that have benefited from the rapid progress in laser technology. And, finally for this section, chapter 22 is devoted to optical and opto-acoustic molecular tomography. It summarizes emerging methods for optical molecular imaging in small animals, mainly in high-performance 
fluorescence visualization in vivo through entire animal bodies and tissues, with the most widely acknowledged technology being the 2008 Nobel-prize-awarded fluorescent protein (FP), able to tag cellular motility and subcellular processes.

Part IV provides extensive coverage of microscopic imaging in research and clinical applications. It stretches from Fourier-domain low-coherence interferometry (chapter 23) and phase imaging microscopy (chapter 24), over confocal microscopy (chapter 25) and fluorescence microscopy (chapter 26), to digital holographic microscopy (chapter 29) and polarized light imaging of biological tissues (chapter 30). All these techniques are introduced systematically, and various applications are discussed, with rich references to recent literature. I was impressed by the depth of presentation of confocal imaging, made easy to understand without loss of detail. Equally exciting was chapter 26 on fluorescence microscopy with structured excitation illumination, where the reader can find background information along with well illustrated examples and application information. I also found very useful to novices the introduction to the Point Spread Function (PSP, box 26.1), and the introduction to Fourier Space and Optical Transfer Function (box 26.2), both of which make the math easier to read. For the prepared mind, chapter 29 on digital holographic microscopy gives basic explanations of the principle of holographic data analysis in the space domain and the restoration of the complex wavefront. Issues like optical transfer function and "electronic focusing" are further discussed, along with the optical setup of a digital holographic microscope. I found this chapter useful and horizon-expanding, although the main applications still fall into the "Further Developments" subsection. And finally, the important concepts of polarized light imaging are introduced in chapter 30. Starting with the physical nature and the formal mathematical description of polarized light (Stokes vector) and optical retarders, the Mueller matrix is introduced, describing transport of polarized light along with the graphical representation of the polarization state of light on the Poincare sphere. These mechanisms are essential for measurements and modeling of systems which cause birefringence (change of the polarization state of light). The applications mentioned, unfortunately, are limited to merely the examination of the skin for detection of skin cancer.

Part V discusses molecular probe development and molecular imaging. Attention is paid to molecular reporter systems - molecular probes that enable specific detection of biochemical signatures in living cells or organisms with only minimal interruption of native physiological processes. Related to this, various modalities are used that vary in spatial resolution and sensitivity (CT, MRI, PET, SPECT etc). Multimodality imaging technologies are discussed, such as combining nuclear or optical imaging with MRI or CT. Multimodal molecular imaging agents, such as the combination of multiple imaging reporters into a single molecule, are discussed, aimed at enhancing efficacy of detection. Further, the use of nanoparticles for targeted therapeutics and diagnostics is introduced. Fundamental optical properties of nanoparticles are discussed, as well as technologies for modification of their surface properties in a manner that makes them appropriate for safe in-vivo application, minimization of toxicity, and optimization of delivery strategies. Finally, chapter 33 is devoted to plasmonic nanoprobes using the displacement of conduction electrons into frequency oscillations.

Part VI is devoted to Phototerapy, highlighting the use of light in disease and injury treatment. The chapter on photodynamic therapy (chapter 34) discusses photochemical 
reactions mediated by a pharmacological or endogenously generated photosensitizer. Intraluminal, dermatological, retinal, and some recent oncological applications are covered. And, finally, chapter 35 presents aspects of low level laser and light therapy in the form of biostimulation. The biological photoresponse as a function of the wavelength is presented, and the role of mitochondria and cellular respiratory chain is elucidated. Issues like Nitric Oxide release, and downstream cellular effects are discussed. Medical applications such as hair regrowth, acne therapy, wrinkle reduction, wound healing, treatment of musculoskeletal disorders, pain relief, treatment of dental complications etc. are cited. A lot of material feeding the curious and creative mind.

This handbook is a successful attempt to provide as much as possible information to a number of physicists, $R \& D$ engineers, clinicians and scientists who would like to apply contemporary concepts and techniques to solving biomedical diagnostic and therapeutic challenges. It often covers areas that are not conventionally addressed, and is generally thought-provoking and brain-stimulating. At the end of each chapter, the reader can find a wealth of information in the form of pertinent references, the majority of which are from the last decade. The book can serve as an excellent tutorial for students and young investigators, finding their way through the intense and exciting field of biomedical optics. It is a relevant reference for scientists, and also a good teaching tool.

The book is far from exhaustive, and probably does not need to be. Yet, in this reviewer's opinion, this edition would have profited from inclusion of further pertinent material, such as the use of voltage sensitive dyes to optically record myocardial excitation and depolarization front propagation - a method that has been used in the last two decades in cardiac electrophysiology research. Indeed, chapter 31 deals with molecular probes, molecular reporter systems and imaging agents, but fluorescence-based visualization of fast changing myocardial transmembrane potentials seem to have been omitted. Another interesting area which might have been beneficial, is retinal birefringence scanning (RBS) - a technique based on the property of the retinal nerve fibers or the Henle fibers to change the polarization state of light. RBS methods have been used in ophthalmology during the last decade to assess eye alignment by locating the fovea (the most sensitive part of the retina), and for measuring the thickness of the retinal nerve fiber layer (RNFL) in glaucoma research and diagnostic instrumentation (such as the GDx from Carl Zeiss, for instance). The ideal place to include such material would have been chapter 30 (Polarized Light Imaging of Biological Tissues), or a separate chapter immediately following chapter 30 .

This book compares favorably to the following recent books covering similar material in the field of Biomedical Optics:

1. Biomedical Optics: Principles and Imaging. Lihong V. Wang, Hsin-i Wu. Wiley, 2007; ISBN-10: 0471743046, ISBN-13: 978-0471743040

2. An Introduction to Biomedical Optics (Series in Optics and Optoelectronics). Robert Splinter, Brett A. Hooper, Taylor and Francis, 2006; ISBN-10: 0750309385, ISBN-13: 978-0750309387.

3. Introduction to Optical Microscopy. Jerome Mertz, Roberts and Company Publishers, 2009, ISBN-10: 0981519482, ISBN-13: 978-0981519487. 
4. Photoacoustic Imaging and Spectroscopy (Optical Science and Engineering). Lihong Wang, CRC Press, Taylor and Francis Group, 2009, ISBN-10: 1420059912, ISBN-13: 978-1420059915.

5. Principles of Fluorescence Spectroscopy. Joseph R. Lakowicz, Springer, 2006, ISBN-10: 0387312781, ISBN-13: 978-0387312781

6. Biomedical Photonics Handbook. Tuan Vo-Dinh (Editor), CRC Press, 2003, ISBN-10: 0849311160, ISBN-13: 978-0849311161

7. Tissue Optics: Light Scattering Methods and Instruments for Medical Diagnosis, Second Edition (SPIE Press Monograph Vol. PM166). Valery Tuchin, The Society of Photo-Optical Instrumentation Engineers (SPIE), 2007, ISBN-10: 0819464333, ISBN-13: 978-0819464330

8. Optical Polarization in Biomedical Applications (Biological and Medical Physics, Biomedical Engineering) Valery V. Tuchin, Lihong Wang, Dmitry A. Zimnyakov, Springer, 2006, ISBN-10: 3540258760, ISBN-13: 978-3540258766

9. Handbook of Optical Coherence Tomography. Brett Bouma (Editor), Marcel Dekker 2001, ISBN-10: 0824705580, ISBN-13: 978-0824705589

10. Optical Coherence Tomography: Principles and Applications. Mark E. Brezinski, Elsevier, 2006, ISBN-10: 0121335704, ISBN-13: 978-0121335700

While some of the above sources are mainly of tutorial value (\#2,\#3), others do offer in-depth knowledge in the subareas covered (\#4, \#5, \#7, \#8, \#10). The present volume is a good balance between introductory knowledge and high-level expertise, without burdening the reader with excessive data. What makes it really unique is its scope. This is a handbook of thirty five chapters, covering an enormous breadth of material, much of which is not included in \#1 - \#10. An additional value is that the book is truly state-of-the-art. With the exception of \#4, all other books have been published at least 4 years before it. In a dynamic field like biomedical optics, this usually makes a difference.

Competing interests

The authors declare that they have no competing interests.

Received: 31 January 2012 Accepted: 10 February 2012 Published: 10 February 2012

doi:10.1186/1475-925X-11-7

Cite this article as: Gramatikov: "Handbook of biomedical optics", edited by David A. Boas, Constantinos Pitris, and Nimmi Ramanujam. BioMedical Engineering OnLine 2012 11:7. 